\title{
Missed Diagnosis of Cholangiocarcinoma Presenting with Atypical Symptoms
}

\author{
Ren Kawamura, Yukinori Harada, Taro Shimizu \\ Department of Diagnostic and Generalist Medicine, Dokkyo Medical University Hospital, Mibu, Tochigi, Japan
}

Received: $10 / 12 / 2020$

Accepted: 02/01/2021

Published: 26/01/2021

How to cite this article: Kawamura R, Harada Y, Shimizu T. Missed diagnosis of cholangiocarcinoma presenting with atypical symptoms. EJCRIM 2021;8: doi:10.12890/2021_002207.

Conflicts of Interests: The Authors declare that there are no competing interests.

This article is licensed under a Commons Attribution Non-Commercial 4.0 License

\section{ABSTRACT}

We report a case of delayed diagnosis of cholangiocarcinoma. A 62-year-old man developed acute abdominal pain in multiple sites. As the distribution pattern of the abdominal pain was not correctly interpreted based on the mechanisms of visceral and referred pain, the patient was not investigated with the best diagnostic test at first presentation. Moreover, miscommunication between physicians in a clinic and separate hospital delayed diagnosis. For prompt diagnosis, physicians should be practice careful reasoning and focus on good communication with physicians outside their hospital.

\section{LEARNING POINTS}

- Abdominal pain without jaundice can be an initial symptom in patients with cholangiocarcinoma.

- Cholangiocarcinoma in the lower common bile duct can present as lower abdominal pain referred through the 7th-11th thoracic nerves.

- Physicians can determine the origin of abdominal pain through correct interpretation of the distribution pattern of abdominal pain based on knowledge of pathophysiology.

\section{KEYWORDS}

Cholangiocarcinoma, visceral pain, referred pain

\section{CASE DESCRIPTION}

A 62-year-old man presented with abdominal pain. Seven days previously, he had developed right lower abdominal pain which moved to the right upper quadrant and then to his back over the next few days. He then attended a clinic where he was prescribed analgesics, with minimal effect. The following day, he called an ambulance at midnight due to acute epigastric pain with worsening of right upper quadrant pain. The pain was intermittent, intensified after eating, and was not correlated with body position or movement. He denied nausea, vomiting, diarrhoea, constipation or melena. His medical history included myocardial infarction, diabetes mellitus, and paroxysmal atrial fibrillation. He was not distressed on arrival at hospital. His body temperature was $37.5^{\circ}$, blood pressure was $162 / 89 \mathrm{mmHg}$, pulse rate was 90 beats per minute, and respiratory rate was 12 breaths per minute. His abdomen was soft and flat, with slight tenderness in the right upper to the right lower quadrants without rebound tenderness or guarding.

\section{Methods and Procedures}

No specific abnormal findings were found on laboratory tests (Table 1). Abdominal ultrasonography showed two gallstones in the gallbladder, but there was no evidence of bile duct dilatation, gallbladder swelling, or intestinal dilatation. The abdominal pain was relieved with intravenous acetaminophen and the patient was sent home. 
Subsequently, however, his epigastric pain slowly became persistent and severe. Four days later, he underwent a lower gastrointestinal series with barium enema at the clinic, which showed normal results. The following day, he visited our hospital again seeking the cause of the abdominal pain. An abdominal CT without contrast was inconclusive because of the barium artifact; however, an abnormal soft tissue mass about $2.0 \mathrm{~cm}$ in diameter was seen on the root of the celiac artery (Fig. 1), which was suspected to be the cause of the pain. A contrastenhanced CT was required for further assessment but had to be postponed until complete excretion of the barium for a clear imaging result. Two days later, the patient underwent scheduled upper gastrointestinal endoscopy at the clinic, which found multiple small superficial erosions on the gastric mucosa, which however were thought to be too small to cause the extreme pain.

Five days later, a contrast-enhanced CT was finally performed and showed wall thickening of the distal bile duct (Fig. 2). Intraductal ultrasonography during endoscopic retrograde cholangiopancreatography demonstrated circumferential wall thickening of the common bile duct, which extended to the intra-pancreatic bile duct. The patient was diagnosed with cholangiocarcinoma following endoscopic ultrasound-fine needle aspiration of the bile duct. His pain was relieved following drainage of the bile duct. The tumour was considered inoperative as it had acutely progressed, and the patient was discharged to palliative care at home.

\begin{tabular}{|l|l|}
\hline White blood cell count & $7,800 / \mathrm{mm}^{3}$ \\
\hline Haemoglobin & $15.8 \mathrm{~g} / \mathrm{dl}$ \\
\hline Platelet count & $27.3 \times 104 / \mathrm{mm}^{3}$ \\
\hline Aspartate aminotransferase & $15 \mathrm{U} / \mathrm{l}$ \\
\hline Alanine aminotransferase & $13 \mathrm{U} / \mathrm{l}$ \\
\hline Lactate dehydrogenase & $152 \mathrm{U} / \mathrm{l}$ \\
\hline Alkaline phosphatase & $396 \mathrm{U} / \mathrm{l}$ \\
\hline Gamma glutamyl transferase & $30 \mathrm{U} / \mathrm{l}$ \\
\hline Total bilirubin & $0.6 \mathrm{mg} / \mathrm{dl}$ \\
\hline Blood urea nitrogen & $20 \mathrm{mg} / \mathrm{dl}$ \\
\hline Creatinine & $0.81 \mathrm{mg} / \mathrm{dl}$ \\
\hline Creatine kinase & $126 \mathrm{U} / \mathrm{l}$ \\
\hline Cardiac troponin I & $0.046 \mathrm{ng} / \mathrm{ml}$ \\
\hline C-reactive protein & $6.9 \mathrm{mg} / \mathrm{l}$ \\
\hline
\end{tabular}

Table 1. Laboratory results at the first visit

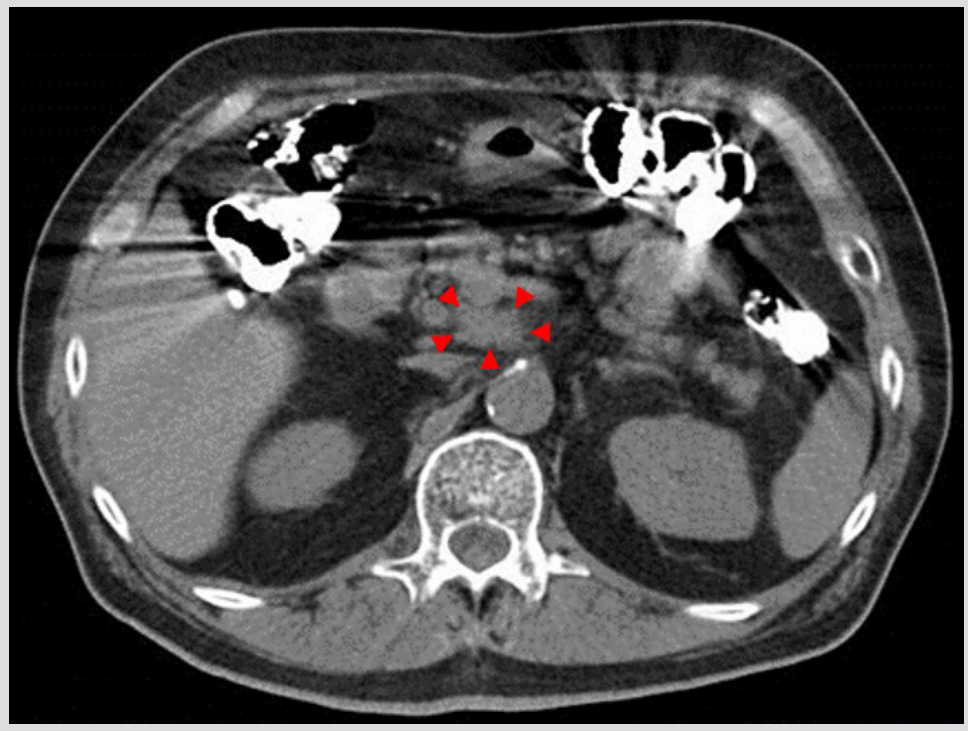

Figure 1. Abdominal CT without contrast showed an inconclusive result due to barium artifact. However, an abnormal soft tissue mass around $2.0 \mathrm{~cm}$ in diameter was detected on the root of the celiac artery

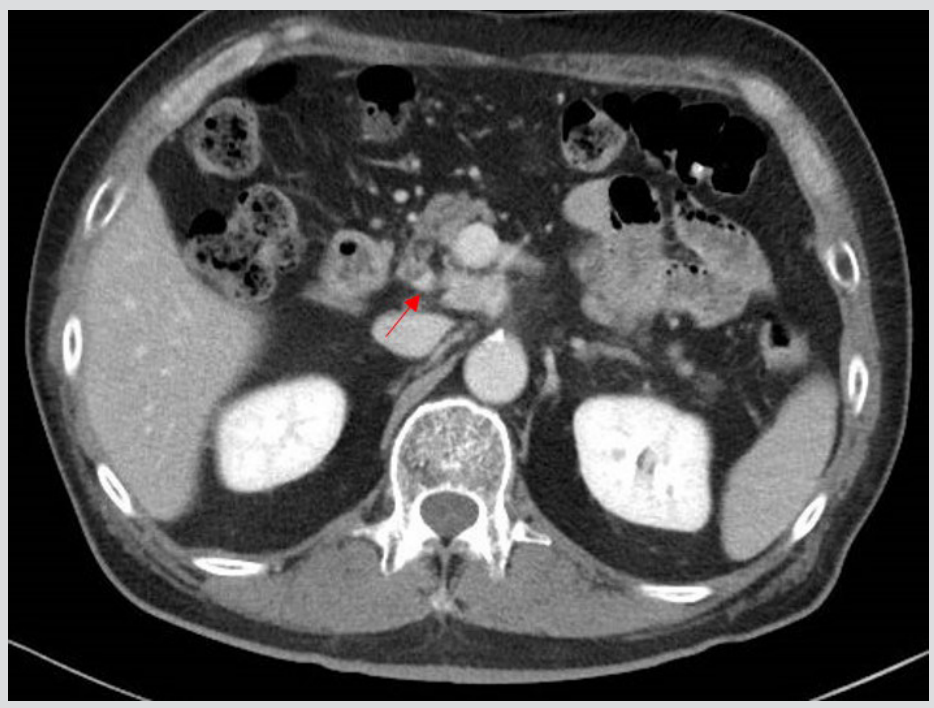

Figure 2. Contrast-enhanced abdominal CT revealed wall thickening of the distal bile duct with a contrast effect in addition to the mass already recognized 


\section{DISCUSSION}

A diagnostic delay occurred in this case, principally due to failure to recognise the cancer for two possible reasons: first, cholangiocarcinoma is very rare and so was not included in the differential diagnosis, and second, this patient presented to the emergency department with acute extreme abdominal pain without jaundice, symptoms that are atypical for cholangiocarcinoma. Jaundice is the initial presentation in over $90 \%$ of patients with extrahepatic cholangiocarcinoma ${ }^{[1]}$.

It is important to correctly interpret the distribution pattern of abdominal pain in order to reach the proper diagnosis. Patients with cholangiocarcinoma usually develop pain in the right upper abdominal quadrant ${ }^{[1]}$. In this case, pain in other locations hindered physicians from making the right clinical interpretation. The vague lower abdominal pain was interpreted as coming from the intestine, which resulted in performance of the endoscopy and barium enema contrast for diagnosis. Retrospectively, however, the epigastric, right lower abdominal quadrant, and back pain may have been referred pain from cholangiocarcinoma in the lower common bile duct through the 7th-11th thoracic nerves ${ }^{[2]}$. In addition, stomach or duodenal disease alone would not have explained the right lower abdominal pain ${ }^{[3]}$, and colonic disease alone would not have caused the back pain in this patient ${ }^{[4]}$. The pain was more intense and sharp in the epigastrium but more vague in the right upper quadrant. Since visceral pain is usually vague, and referred pain is usually sharp and intense ${ }^{[5]}$, this information should have suggested that the principal lesion was in an organ in the right upper quadrant. Also, upon physical examination, no peritoneal irritation other than mild tenderness was observed, suggesting that the lesion was not in contact with the peritoneum. All things considered, it should have been suspected that the chief lesion was in the bile tract, pancreas or duodenum. Finally, if doctors had taken a wider overall view and considered the causes of severe back pain, metastatic bile duct carcinoma may have been included as a differential diagnosis during the initial visit and contrast-enhanced CT could have been performed at an earlier stage.

In this case, communication errors between physicians working at the clinic and the hospital delayed diagnosis. If they had communicated properly, the patient would not have received a barium enema, and the contrast-enhanced CT could have been carried out earlier. In conclusion, doctors should identify the exact origin of abdominal pain in order to make a correct and timely diagnosis. They should also schedule evaluation tests properly.

\section{REFERENCES}

1. Khan AS, Dageforde LA. Cholangiocarcinoma. Surg Clin North Am 2019;99:315-335.

2. Doran FS. The sites to which pain is referred from the common bile-duct in man and its implication for the theory of referred pain. Br J Surg 1967;54:599-606.

3. Drewes AM, Arendt-Nielsen L, Jensen JH, Hansen JB, Krarup HB, Tage-Jensen U. Experimental pain in the stomach: a model based on electrical stimulation guided by gastroscopy. Gut 1997;41:753-757.

4. Gebhart GF, Bielefeldt K. Physiology of visceral pain. Compr Physiol 2016;6:1609-1633.

5. Sikandar S, Dickenson AH. Visceral pain: the ins and outs, the ups and downs. Curr Opin Support Palliat Care 2012;6:17-26. 